\title{
Drugs Highly Associated with Infusion Reactions Reported using Two Different Data-mining Methodologies
}

Philip W Moore ${ }^{1 *}$, Keith K Burkhart ${ }^{1-3}$ and David Jackson ${ }^{4}$

${ }^{1}$ PinnacleHealth, Department of Internal Medicine, Harrisburg, PA, USA

${ }^{2}$ Pennsylvania State University College of Medicine, Department of Emergency Medicine, Hershey, PA, USA

${ }^{3}$ Food and Drug Administration, Center for Drug Evaluation and Research, Office of Translational Sciences, Office of Clinical Pharmacology, Silver Spring, MD, USA ${ }^{4}$ Molecular Health $\mathrm{GmbH}$, Heidelberg, Germany

\begin{abstract}
Objective: Infusion reactions can be serious life threatening adverse events and have been associated with many drugs and biologic agents. Our objective was to report drugs associated with infusion reactions using two different data-mining methodologies.

Methods: The Food and Drug Administration Adverse Event Reporting System (FAERS) was data-mined for drugs highly associated with infusion reactions. Drugs were included if there were $>10$ reported adverse events and if the Empirical Bayesian Geometric Mean (EBGM) score $\geq 2$. Molecular Health's MASE (Molecular Analysis of Side Effects) reports Proportional Reporting Ratios (PRR) for drugs highly associated with infusion reactions and was cross-referenced to improve detection sensitivity.

Results: Using FAERS, the highest EBGM scores by class were reported as: pegloticase and $\alpha-1$ antitrypsyn (enzymes), iron dextran and ferric gluconate (electrolytes and nutrients), infliximab and gemtuzumab (immunomodulators), and paclitaxel and oxaliplatin (antimetabolites). Using MASE, the highest PRR scores were reported as: idursulfase and galsulfase (enzymes), iron dextran and phytonadione (electolytes and nutrients), gemtuzumab and infliximab (immunomodulators), mercaptopurine and azathioprine (antimetabolites). Amphotericin and vancomycin had the highest scores for the antimicrobial class for both FAERS and MASE.

Conclusions: Using the two statistical methods EBGM and PRR, both specificity and sensitivity were preserved. However, neither system detected several drugs with established relationships to infusion reactions, including protamine and nitroglycerine. Reactions caused by these drugs were possibly underreported because the effects have been well established or due to evolution of administration with slower administration. We hope this analysis encourages further research into overlapping mechanisms for infusion reactions.
\end{abstract}

Keywords: Data mining; Adverse event reporting system; Molecular analysis of sideeffects; Infusionreactions; Histamine release; Cytokine release; Trans-nitrosylation; S-nitrosylation nitric oxide; Nitric oxide synthase; Guanylatecyclase reactive oxygen species; Reactive nitrogen species; Peroxynitrate

Abbreviations: EBGM: Empirical Bayesian Geometric; FDA: Food and Drug Administration; FAERS: Food and Drug Administration Adverse Event Reporting System; IG: Immunoglobulin; MASE: Molecular Analysis of Side Effects; NO: Nitric Oxide; NOS: Nitric Oxide Synthase; PRR: Proportional Reporting Ratio; ROS: Reactive Oxygen Species; RNS: Reactive Nitrogen Species; TNF- $\alpha$ : Tumor Necrosis Factor- $\alpha$

\section{Background}

Infusion reactions may mimic drug hypersensitivity, with a spectrum of variability and heterogeneity for both individual and drug; symptoms may include anxiety, diaphoresis, rigors/chills, fever, pruritus, urticaria, angioedema, headache, nausea, emesis, diarrhea, chest pain, dyspnea, wheezing/bronchospasm, hypoxia, respiratory failure, hypotension, and death [1-4]. Symptoms usually occur shortly after the infusion begins but can have delayed onset [1-4]. Symptoms may decrease when the infusion rate is discontinued or slowed; at other times, the symptoms persist [1-4]. To add to the complexity surrounding infusion reactions, multiple nomenclatures are used, including drugmediated hypersensitivity, infusion-related toxicity, cytokine storm, cytokine-release syndrome, anaphylactoid reaction, and serum sickness-like illness [2,4-8]. The spectrum of symptoms, variable onset, and the multiple names implicate the immune system. Recognizing the molecular and ontologic mayhem surrounding infusion reactions, we used software technology to investigate drugs highly associated with infusion reactions.

Advances in information technology allow data integration at many levels; molecular pathways can be visualized through dependency graphs and statistical modeling [9]. MASE (Molecular Analysis of Side Effects) was developed by Molecular Health to connect biomolecules, pathways, targets and diseases in a drug-centric manner. Drugs highly associated with infusion reactions are reported by MASE using Proportional Reporting Ratios (PRRs). PRRs are calculated by the ratio of the observed to the expected number of drug-event pairs. The Empirical Bayesian Geometric Mean (EBGM) value is the PRR adjusted

${ }^{*}$ Corresponding author: Philip Moore DO, Medical Toxicologist and Hospitalist, Department of Internal Medicine, Pinnacle Health, Brady Building, Suite 6B, 111 S. Front St., Harrisburg, PA 17104, USA, Tel: (717)547-6234; Fax: (717)547-6235; E-mail: pwmoore@pinnaclehealth.org

Received December 04, 2013; Accepted January 25, 2014; Published January 28,2014

Citation: Moore PW, Burkhart KK, Jackson D (2014) Drugs Highly Associated with Infusion Reactions Reported using Two Different Data-mining Methodologies. J Blood Disorders Transf 5: 195. doi: 10.4172/2155-9864.1000195

Copyright: (c) 2014 Moore PW, et al. This is an open-access article distributed under the terms of the Creative Commons Attribution License, which permits unrestricted use, distribution, and reproduction in any medium, provided the original author and source are credited. 
for differences in reporting rates while stratifying the expected rates by variables within the dataset $[10,11]$. Using data dating back to 1960 from voluntarily submitted reports from physicians and pharmacists, the Food and Drug Administration's (FDA) Adverse Event Reporting System (FAERS) uses EBGM to report the signal strength for drugs, and their adverse effects. Each reporting ratio shrinks towards one; this conservative approach considers EBGM and PRR values $\geq 2.0$ to be the safety signal threshold. Higher values denote a stronger association between the drug and reported adverse drug reactions (ADRs) [10,11]. Data mining and statistical analysis with PRRs have enhanced postmarketing pharmacovigilance [12-15]. By using both databases, two different data mining algorithms can be compared. Previous studies have demonstrated PRRs to be more sensitive, while EBGM more specific $[10,16]$. Infusion reaction homology and heterogeneity can be simultaneously queried using both MASE and the FAERS databases to improve signal visualization and detection.

\section{Methods}

MASE and FAERS data were queried on the same day, which allowed validation of MASE against a larger dataset. Public FAERS data was available from 2004 to present; internal Food and Drug Administration data was available from 1960. The preferred search term was "infusion reaction" and both databases use the Medical Dictionary for Regulatory Activities Preferred Terms coding scheme. MASE reported the PRR score and number of ADRs, pathways, targets, diseases and outcomes. The FAERS database reported the EBGM score, number of ADRs, diseases and outcomes. Using both systems, we filtered the identified drugs, using a threshold of EBGM or PRR $\geq 2$, and number of ADR reports $>10$. Requiring $>10$ ADR reports is an extra level of signal strength beyond requiring only $E B G M \geq 2$. Each drug's EBGM and PRR were plotted alongside its $90 \%$ confidence interval and drugs with intervals $<2$ were excluded.

MASE collects demographic information including signs and symptoms, co-morbidities, pathways, and outcomes. Resulting drugs were grouped by mechanism. Since neither MASE nor FAERS differentiated a drug's labeled indication and/or concomitant presence, the investigator's knowledge of clinical and pharmacologic drug activity was applied to find exclusions.

MASE generated dependency graphs, with concurrent visualization of both drug and infusion reactions' overlapping molecular targets and pathways. MASE was used to offer explanations for protective factors such as higher serum acetaminophen concentration, or pretreatment with histamine blocker or corticosteroid [3].

The resultant drugs were grouped based on drug classification and discussed in the order of decreasing EBGM as determined by the mean score. Using the PubMed database, the English language literature was searched to determine common mechanisms for infusion reactions within drug classifications and for their indication.

\section{Results}

FAERS was queried on March 14, 2013 and data-mining resulted in 89 drugs with $E B G M \geq 2$, of which 45 drugs had $>10$ ADRs. Two drugs were excluded, based on the investigator's perceived indication (Table 1). The remaining 43 drugs were ranked by descending EBGM and were graphed with their $90 \%$ confidence interval and number of ADR reports (Figure 1). The bars on the graph are colored based on similar drug classification. There were 14 drugs classified as immunomodulators (red), 12 drugs as enzymes (purple), 6 drugs as antimetabolites (green),

\begin{tabular}{|c|c|}
\hline MASE & FAERS \\
\hline Bepotastine & Sodium chloride \\
\hline almitrine & Hydrocortisone \\
\hline Diphenhydramine & \\
\hline Palonosetron & \\
\hline Chlorpheniramine & \\
\hline Dimethindene & \\
\hline Clemastine & \\
\hline Hydrocortisone & \\
\hline Dolasetron & \\
\hline Mesalazine & \\
\hline Dimenhydrinate & \\
\hline Salsalate & \\
\hline Methylprednisolone & \\
\hline Cimetidine & \\
\hline Granisetron & \\
\hline Dexamethasone & \\
\hline Ondansetron & \\
\hline Prednisolone & \\
\hline Sulfasalazine & \\
\hline Acetaminophen & \\
\hline
\end{tabular}

Table 1: Excluded drugs. MASE (Molecular Analysis of Side Effects) and FAERS (FDA's Adverse Event Reporting System) drugs associated with infusion reactions were excluded if their presence was attributed to the symptomatic treatment of infusion reactions.

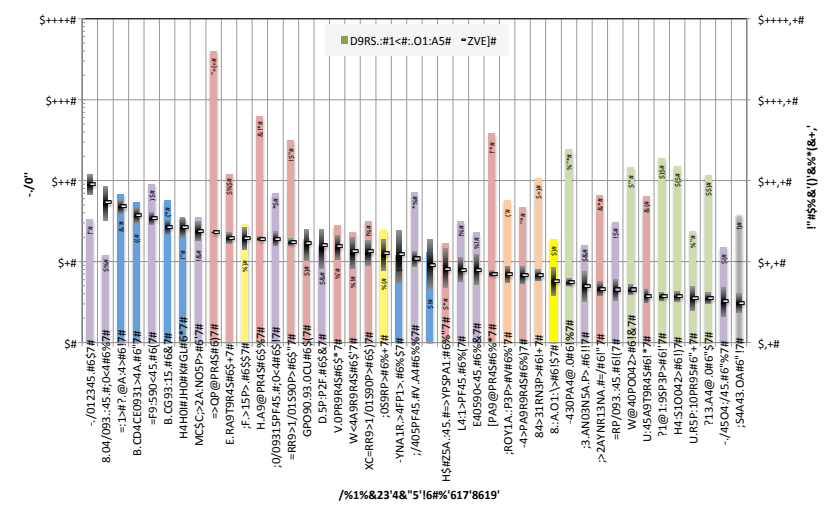

Figure 1: Drugs associated with infusion reaction are ranked by descending EBGM and number of reports using FAERS.

6 drugs as electrolytes (blue), 2 drugs as antibiotics or antifungals (orange), and 3 drugs as other (yellow).

MASE was queried on March 14, 2013. There were 8198 reported infusion reactions from 2003 to mid-2011 and the top 15 indications, reactions, molecular target and mechanisms are defined in Table 2. MASE identified 861 drugs associated with the search term "infusion reaction," 60 had both a $P R R \geq 2$ and $>10$ ADR reports. Twenty drugs were excluded as likely being present to treat the symptoms of the infusion reaction (Table 1). The 40 remaining drugs were ranked by descending PRR and were graphed with their $90 \%$ confidence interval and number of associated reports (Figure 2). The bars on the graph are colored based on similar drug classification. There were 13 drugs classified as antimetabolites (green), 9 drugs as immunomodulators (red), 6 antibiotics or antifungals (orange), 5 drugs were classified as enzymes (purple), 3 drugs as electrolytes (blue), and 4 drugs as other (yellow). The FAERS and MASE drugs were listed alphabetically within their drug classes to compare internal rank, EBGM, and PRR in Table 3. 


\begin{tabular}{|c|c|c|c|c|}
\hline \multicolumn{2}{|r|}{ Indication } & Reactions & Molecular target & Molecular Mechanism \\
\hline 1 & mucopolysaccaridosis type II & red man syndrome & heparan sulfate & Golgi associated vesicle biogenesis \\
\hline 2 & hypogammaglobulinemia & neutralizing antibodies positive & dermatan sulfate & Alternative complement activation \\
\hline 3 & mucopolysaccaridosis & infusion site pruritus & $\begin{array}{c}\text { mannose-6-phosphate receptor bind- } \\
\text { ing protein } 1\end{array}$ & Initial triggering of complement \\
\hline 4 & mucopolysaccaridosis type VI & $\begin{array}{c}\text { no reaction on previous exposure to } \\
\text { drug }\end{array}$ & ferritin heavy chain & Alpha-defensins \\
\hline 5 & glycogen storage disease type II & infusion site erythema & ferritin light chain & $\begin{array}{l}\text { Localization of the pinch-ilk-parvin complex } \\
\text { to focal adhesions }\end{array}$ \\
\hline 6 & $\begin{array}{l}\text { chronic inflammatory demyelinating } \\
\text { polyradiculoneuropathy }\end{array}$ & oxygen saturation decreased & myeloid cell surface antigen cd33 & Other semaphorin interactions \\
\hline 7 & alpha-1-antitrypsin deficiency & blood pressure immeasurable & globotriaosylceramide & Arf1 pathway \\
\hline 8 & immunodeficiency & anaphylactoid reaction & serotransferrin & $\begin{array}{l}\text { Pten dependent cell cycle arrest and } \\
\text { apoptosis }\end{array}$ \\
\hline 9 & immunodeficiency common variable & sense of oppression & hemoglobin subunit alpha & $\begin{array}{l}\text { Hiv-1 defeats host-mediated resistance by } \\
\text { cem } 15\end{array}$ \\
\hline 10 & mucopolysaccharidosis type I & blood immunoglobulin g increased & complement c3 & Hemoglobins chaperone \\
\hline 11 & iron deficiency anemia & incorrect drug administration rate & complement c4a & Foxo family signaling \\
\hline 12 & Fabry's disease & cyanosis & complement c4b & $\begin{array}{l}\text { Double stranded ma induced gene expres- } \\
\text { sion }\end{array}$ \\
\hline 13 & pyodermagangrenosum & infusion site swelling & $\begin{array}{l}\text { high affinity immunoglobulin gamma } \\
\text { fc receptor 1b }\end{array}$ & endogenoustlr signaling \\
\hline 14 & uterine cancer & type IV hypersensitivity reaction & $\begin{array}{l}\text { low affinity immunoglobulin gamma fc } \\
\text { region receptor } 2 a\end{array}$ & Ikk complex recruitment mediated by rip 1 \\
\hline 15 & head and neck cancer & suffocation feeling & $\begin{array}{l}\text { low affinity immunoglobulin gamma fc } \\
\text { region receptor } 2 c\end{array}$ & Irak1 recruits ikk complex \\
\hline
\end{tabular}

Table 2: Demographics of infusion reactions when $P R R>2$ and $N>10$.

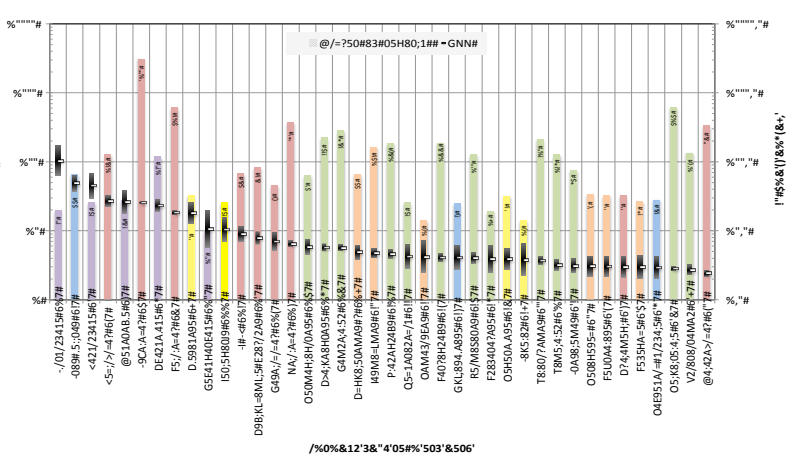

Figure 2: Drugs associated with infusion reaction are ranked by descending PRR and number of reports using MASE.

\section{Discussion}

\section{Electrolytes and nutrients}

The drugs classified as electrolytes and nutrients had the highest overall EBGM scores, with a mean EBGM score of 30.8. Iron products represented 3 of the top 6 drugs associated with FAERS infusion reactions while iron dextran appeared in the top two drugs associated with MASE infusion reactions. Fourteen percent of drugs associated with infusion reactions on the FAERS list were classified as electrolytes and nutrients which was higher than the $7.5 \%$ of drugs reported by MASE. This difference likely reflects prescribing trends since MASE data is obtained from public data starting in 2004 while FAERS starts in the 1960s. Iron products have long been associated with infusion reactions and comparative studies have demonstrated that iron sucrose and ferric gluconate have the lowest rates of infusion reactions and iron dextran the highest [17]. Ferric gluconate and iron sucrose are listed respectively on FAERS as number 3 and 6 overall.
The association between iron and infusion reactions could be through nitric oxide (NO), an important member of a free radical buffer system present in low concentrations to delicately balance endothelial smooth muscle tone [5,6,18-21]. NO nitrosylates transitional metals with a higher affinity than for oxygen or carbon monoxide and has a higher affinity for ferric iron (II) than for ferrous iron (III) [21]. The bound NO group has increased electrophilicity or nucleophilicity and can disrupt cellular signaling (subunits of hemoglobin and methemoglobin) and mitochondrial respiration, (cytochrome C) and increase NO availability and release thereby resulting in conditions favoring an infusion reaction [21]. The affinity of NO for transition metals with subsequent increased reactivity may explain why other metals, such as magnesium, also appear on the list of drugs with high EBGM.

\section{Enzymes}

Drugs classified as enzymes had a mean EBGM score of 22.7 and were more prevalent within FAERS (27.9\%) than MASE (12.5\%). The most common indication for these enzymes was glycogen storage disease; 7 of 12 FAERS drugs classified as enzymes have indications for glycogen storage diseases as compared to 3 of 5 from MASE. Six of the 15 most common indications for drugs from MASE associated with infusion reactions were for glycogen storage disease (Table 2). Glycogen storage diseases may increase susceptibility to infusion reactions by increasing levels of cytokines [2,22]. Many of these cytokines, mediate symptoms of an infusion reaction by signal amplification, histamine release, and activation of nitric oxide synthase (NOS) each of which increase NO release [5,6].

Another drug classified as an enzyme, pegloticase, catalyzes the elimination of uric acid and is indicated for the treatment of hyperuricemia related to gout and/or tumor lysis syndrome. Pegloticase was the FAERS drug most associated with infusion reactions but did not rank among MASE drugs. Gout may be a condition predisposed toward infusion reactions because of increased concentrations of reactive nitrogen species such as peroxynitrite [23]. Peroxynitrate, is 
Citation: Moore PW, Burkhart KK, Jackson D (2014) Drugs Highly Associated with Infusion Reactions Reported using Two Different Data-mining Methodologies. J Blood Disorders Transf 5: 195. doi: 10.4172/2155-9864.1000195

Page 4 of 6

\begin{tabular}{|c|c|c|c|c|c|c|c|}
\hline \multirow[t]{2}{*}{ Classification } & \multirow[t]{2}{*}{ Generic name } & \multicolumn{3}{|c|}{ FAERS } & \multicolumn{3}{|c|}{ MASE } \\
\hline & & Rank & $\mathbf{N}$ & EBGM & Rank & $\mathbf{N}$ & PRR \\
\hline Analgesic & Meperidine & $\mathrm{N} / \mathrm{A}$ & $\mathrm{N} / \mathrm{A}$ & N/A & 28 & 32 & 3.9 \\
\hline AADS & Adenosine & 11 & 29 & 19.4 & 9 & 33 & 18.0 \\
\hline ABX, Beta-lactam & Meropenem & N/A & $\mathrm{N} / \mathrm{A}$ & $\mathrm{N} / \mathrm{A}$ & 28 & 34 & 3.1 \\
\hline ABX, Cephalosporin & Cefepime & N/A & 9 & 2.1 & 36 & 27 & 3.0 \\
\hline ABX, Cephalosporin & Ceftriaxone & N/A & 20 & 1.3 & 34 & 33 & 3.1 \\
\hline ABX, Glycopeptide & Vancomycin & 30 & 109 & 6.8 & 20 & 162 & 4.7 \\
\hline AFP, Echinocandin & Micafungin & N/A & 6 & 1.9 & 23 & 14 & 4.2 \\
\hline AFP, Polyene & Amphotericin B & 28 & 58 & 7.0 & 19 & 66 & 4.9 \\
\hline ANTI & Leucovorin & N/A & $\mathrm{N} / \mathrm{A}$ & N/A & 26 & 130 & 4.0 \\
\hline ANTI & Methotrexate & $\mathrm{N} / \mathrm{A}$ & 80 & 1.9 & 38 & 616 & 2.8 \\
\hline ANTI, ANT & Doxorubicin & 38 & 191 & 3.7 & 30 & 210 & 3.7 \\
\hline ANTI, Kinase Inh & Temsirolimus & 40 & 24 & 3.5 & 22 & 26 & 4.2 \\
\hline ANTI, Pt & Carboplatin & 39 & 151 & 3.7 & 24 & 188 & 4.1 \\
\hline ANTI, Pt & Oxaliplatin & 36 & 148 & 4.5 & 21 & 184 & 4.6 \\
\hline ANTI, pur & Azathioprine & $\mathrm{N} / \mathrm{A}$ & $\mathrm{N} / \mathrm{A}$ & N/A & 17 & 226 & 5.8 \\
\hline ANTI, pur & Clofarabine & $\mathrm{N} / \mathrm{A}$ & 10 & 1.3 & 27 & 19 & 3.9 \\
\hline ANTI, pur & Mercaptopurine & $\mathrm{N} / \mathrm{A}$ & 12 & 1.4 & 16 & 63 & 5.9 \\
\hline ANTI, pyr & Fluorouracil & $\mathrm{N} / \mathrm{A}$ & 52 & 1.4 & 39 & 134 & 2.7 \\
\hline ANTI, Taxanes & Docetaxel & 41 & 119 & 3.5 & 31 & 127 & 3.2 \\
\hline ANTI, Taxanes & Paclitaxel & 32 & 247 & 5.6 & 18 & 287 & 5.7 \\
\hline ANTI, TOPO Inh & Irinotecan & $\mathrm{N} / \mathrm{A}$ & 33 & 1.6 & 32 & 76 & 3.1 \\
\hline Contrast & lohexol & $\mathrm{N} / \mathrm{A}$ & 11 & 2.3 & 29 & 14 & 3.8 \\
\hline Enz & Acetylcysteine & 33 & 16 & 5.0 & $\mathrm{~N} / \mathrm{A}$ & 25.0 & 2.8 \\
\hline Enz, CHF & Nesiritide & 16 & 16 & 15.9 & 5 & 28 & 26.3 \\
\hline Enz, Gout & Pegaspargase & 42 & 15 & 3.2 & 10 & 10 & 10.6 \\
\hline Enz, Gout & Pegloticase & 1 & 34 & 90.4 & N/A & N/A & $\mathrm{N} / \mathrm{A}$ \\
\hline Enz, GSD & Agalsidase Beta & 22 & 72 & 10.9 & 7 & 120 & 23.3 \\
\hline Enz, GSD & Alglucosidase Alfa & 13 & 71 & 19.2 & $\mathrm{~N} / \mathrm{A}$ & 2.0 & 52.9 \\
\hline Enz, GSD & Galsulfase & 26 & 23 & 7.9 & 3 & 26 & 45.0 \\
\hline Enz, GSD & Idursulfase & 5 & 91 & 34.5 & 1 & 20 & 103.8 \\
\hline Enz, GSD & Imiglucerase & 35 & 31 & 4.5 & $\mathrm{~N} / \mathrm{A}$ & 1.0 & 12.6 \\
\hline Enz, GSD & Laronidase & 25 & 32 & 7.9 & $\mathrm{~N} / \mathrm{A}$ & 3.0 & 48.5 \\
\hline Enz, GSD & Velaglucerase Alfa & 2 & 12 & 54.0 & N/A & N/A & N/A \\
\hline Enz, Pls & $\alpha-1-A n t i t r y p \sin$ & 8 & 36 & 24.0 & $\mathrm{~N} / \mathrm{A}$ & N/A & N/A \\
\hline FEN & $\mathrm{CaCl}$ and $\mathrm{KCl}$ & 7 & 34 & 26.6 & N/A & 8.0 & 1.7 \\
\hline FEN & Phytomenadione & 21 & 12 & 12.4 & 25 & 25 & 4.1 \\
\hline FEN, Fe & Ferric Na Gluc & 4 & 55 & 37.6 & N/A & N/A & N/A \\
\hline FEN, Fe & Ferumoxytol & 23 & 13 & 9.2 & $\mathrm{~N} / \mathrm{A}$ & N/A & $\mathrm{N} / \mathrm{A}$ \\
\hline FEN, Fe & Iron Dextran & 3 & 68 & 48.1 & 2 & 66 & 48.9 \\
\hline FEN, Fe & Iron Sucrose & 6 & 57 & 27.0 & $\mathrm{~N} / \mathrm{A}$ & N/A & $\mathrm{N} / \mathrm{A}$ \\
\hline FEN, Mg & Mg sulfate & N/A & 6 & 3.2 & 37 & 28 & 2.9 \\
\hline IMiD & C1 Esterase Inhibitor (Human) & 24 & 17 & 8.0 & N/A & N/A & $\mathrm{N} / \mathrm{A}$ \\
\hline IMiD & Sipuleucel-T & 15 & 19 & 17.0 & $\mathrm{~N} / \mathrm{A}$ & N/A & $\mathrm{N} / \mathrm{A}$ \\
\hline IMiD, HMAB & Belimumab & 17 & 28 & 15.5 & N/A & N/A & $\mathrm{N} / \mathrm{A}$ \\
\hline IMiD, HMAB & Ofatumumab & 18 & 23 & 13.5 & $\mathrm{~N} / \mathrm{A}$ & N/A & $\mathrm{N} / \mathrm{A}$ \\
\hline IMiD, HMAB & Panitumumab & 29 & 47 & 6.8 & 14 & 45 & 7.1 \\
\hline IMiD, IG & Abatacept & 43 & 39 & 3.1 & 35 & 33 & 3.0 \\
\hline IMiD, IG & $\lg$ & 14 & 314 & 17.4 & 12 & 68 & 9.0 \\
\hline IMiD, IG & Rho-lg & 19 & 32 & 13.3 & $\mathrm{~N} / \mathrm{A}$ & N/A & $\mathrm{N} / \mathrm{A}$ \\
\hline IMiD, MCABS, Chimeric & Cetuximab & 12 & 637 & 19.3 & 8 & 612 & 18.4 \\
\hline IMiD, MCABS, Chimeric & Infliximab & 9 & 4050 & 23.1 & 6 & 3100 & 25.7 \\
\hline IMiD, MCABS, Chimeric & Rituximab & 27 & 387 & 7.1 & 15 & 373 & 6.5 \\
\hline IMiD, MCABS, Humanized & Gemtuzumab & 10 & 121 & 19.7 & 4 & 128 & 27.0 \\
\hline IMiD, MCABS, Humanized & Natalizumab & N/A & 374 & 1.7 & 40 & 338 & 2.4 \\
\hline IMiD, MCABS, Humanized & Trastuzumab & 37 & 65 & 3.8 & $\mathrm{~N} / \mathrm{A}$ & 8.0 & 1.5 \\
\hline IMiD, RMAB & Antithymocytelg & 34 & 67 & 4.6 & 13 & 82 & 7.9 \\
\hline Other & Albumin & 20 & 25 & 12.7 & $\mathrm{~N} / \mathrm{A}$ & $\mathrm{N} / \mathrm{A}$ & N/A \\
\hline Photosensitization & Verteporfin & 31 & 19 & 5.7 & 11 & 26 & 10.5 \\
\hline
\end{tabular}

Abbreviations: AADS: Antiarrhythmic Drugs; AXB: Antibiotic; AFP: Antifungal; ANTI: Antimetabolite; ANT: Anthracycline; Ca: Calcium; Cl: Chloride; CHF: Congestive Heart Failure; Enz: Enzyme; FEN: Fluids/Electolytes/Nutrition; Gluc: Gluconate; GSD: Glycogen Storage Disease; HMAB: Human Monoclonal Antibodies; IG: Immunoglobulin; inh: Inhibitor; IMiD: Immunomodulatory Drug; Fe: Iron; Mg: Magnesium; Min: Mineral; MCABS: Monoclonal Antibodies; MMAB: Mouse Monoclonal Antibodies; Pt: Platinum; K: Potassium; Pls: Proteinase Inhibitor; PKI: Protein Kinase Inhibitor; pur: Purine; pyr: Pyrimidine; Na: Sodium; TOPO: Topoisomerase

Table 3: Drugs highly associated with infusion reactions from MASE (PRR $>2.0$ and N $>10$ reports) and FAERS (EBGM $>2.0$ and N $>10$ reports) are grouped by drug class and then displayed with rank as well as comparison data. EBGM is from FAERS and PRR is displayed from MASE as a statistical comparison and to improve the sensitivity of drug detection. 
an important buffer for $\mathrm{NO}$ and helps to regulate vascular tone; sudden changes can disrupt $\mathrm{NO}$ concentrations and drastically impact vascular tone $[21,23,24]$. Alpha-1-antitrypsin, was classified as an enzyme and ranked eighth on the overall list of FAERS drugs most associated with infusion reactions. Alpha-1-antitrypsincan is linked to infusion reactions as it inhibits NO production and suppresses cytokine release [25].

$\mathrm{N}$-acetylcysteine (NAC) is an enzyme used for regenerating depleted glutathione stores for patients with acetaminophen poisoning and has a well-established relationship with infusion reactions through direct histamine release and increasing prostaglandin-F2- $\alpha[3,4,26,27]$. Other mechanism for NAC contributing to infusion reactions is through S-nitrosylation. NAC contains a thiol (thiolate anion), which can covalently bind NO in a process named protein S-nitrosylation; $\mathrm{S}$-nitrosylation has been associated with the regulation of NO release from erythrocytes $[18,20]$. A prospective study found that up to $60 \%$ of patients receiving NAC experienced symptoms of infusion reactions when administered at a relatively fast rate $(150 \mathrm{mg} / \mathrm{kg}$ NAC in 200 $\mathrm{ml}$ solution, infused over $15 \mathrm{~min}$ ) [3]. Despite this, NAC ranked 33 on FAERS enzymes associated with infusion reactions and was not detected by MASE which could reflect underreporting or prevention through medical knowledge that slowing the infusion rate decreases the incidence and/or severity of infusion reaction symptoms [27]. This may be true for other drugs associated with infusion reactions and offer an explanation why the database with an older dataset (FAERS) differs from the database with the newer dataset (MASE).

\section{Immunomodulators}

Immunomodulators scored high and were prevalent in both FAERS and MASE databases with a prevalence of $32.6 \%$ and $22.5 \%$, respectively (Figures 1 and 2). Immunomodulators have the ability to affect cytokine and histamine release, which impacts NO signaling through iNOS located in macrophages and/or endothelium $[5,6]$. Of the immunomodulators, chimeric monoclonal antibodies scored higher than humanized. Rituximab targets CD20 molecule on B-Cell lymphocytes and causes cytokine release $[1,28]$. Other immunomodulators affecting cytokine release include gemtuzumab, by targeting CD33, cetuximab and panitumumab target epidermal growth factor receptor, [28,29] while trastuzumab targets HER2 receptors $[1,28]$. Rituximab and trastuzumab may carry a higher risk for infusion reaction and structure may be a factor [30]. Immunomodulators are large proteins, chains of amino acids containing thiol groups and/or transitional metals; NO covalently binds sulfur and transitional metals in a process named protein S-nitrosylation or nitrosylation, respectively $[18,31]$. The bound NO group has increased electrophilicity or nucleophilicity and can disrupt cellular signaling, mitochondrial respiration and increase NO availability and release thereby resulting in conditions favoring an infusion reaction [21]. The relative content of sulfur, iron and other transitional metals may be relatively higher for immunomodulators with stronger association for infusion reactions.

\section{Antimicrobials}

Antimicrobials were the drug class composed of antifungals and antibiotics and had a mean EBGM score of 6.9. Vancomycin and amphotericin appeared in both databases (Figures 1 and 2). Micafungin, meropenem, ceftriaxone and cefepime appeared only within MASE, which we suspect is because these are newer antimicrobials as compared to vancomycin and amphotericin. Vancomycin and amphotericin can cause an infusion reaction by increasing histamine release from the endothelium and/or mast cells, directly increasing the release of NO via histamine receptor type 1 or directly effecting vasodilation via histamine receptor type 2 [32-35]. Amphotericin increases the release of superoxide anions from macrophages which react with other reactive oxygen and nitrogen species such as NO resulting in endothelial damage [33]. In the presence of an infection the body responds by producing cytokines and other inflammatory markers; an antibiotic decreases the microbial burden resulting in a relative surplus of cytokines and inflammatory markers. The surplus of cytokines, activate NOS and causes histamine release, which through histamine receptors results in NO release $[5,6]$. This may be one of the mechanisms for which any antimicrobial could predispose an individual for infusion reactions. Cytokine release results in increased inflammatory cell production and migration, which increases the release of histamine [6].

\section{Antimetabolites}

There were more than twice the numbers of antimetabolites found in MASE versus FAERS data with a prevalence of $32.5 \%$ and $14 \%$ respectively (Figures 1 and 2). Nucleotide analogues and topoisomerase inhibitors are the subgroups within drugs classified as antimetabolites which seemed exclusively present in data from MASE. The reason for this is unclear. Other subgroups present in both datasets included anthracyclines, kinase inhibitors, platinum compounds, and taxanes, thought the internal ranking seemed to be variable. Doxorubicin disrupts NO regulation and results in the formation of peroxynitrate [36]. Potent free radicals such as peroxynitrate can react with proteins and alter their structure, function and lifespan $[18,19,21,37]$. Paclitaxel increases production of NO by increasing release of TNF- $\alpha$, which impacts NO signaling through NOS $[5,6,38]$. Cytokine release results in increased inflammatory cell production and migration, which increase the release of histamine [6]. Some drugs such as paclitaxel are packaged in cremophor (polyexalated castor oil) which causes histamine release [39]. Histamine is released from endothelium or mast cells; if the source is mast cells, tryptase will be present $[26,27,35]$. Histamine directly increases the release of NO via histamine receptor type $1[32,34]$. Histamine receptor type 2 has a direct effect on vasodilation via vascular smooth muscle [32]. Thus, histamine directly causes vasodilation via histamine receptor type 2 and indirectly through histamine receptor type 1 . Histamine and $\mathrm{NO}$ are interconnected in many of the reactions and molecular targets and mechanisms from Table $3[5,28,40,41]$.

\section{Limitations}

To strengthen our statistical filter, false positives, which are inherent to data mining methods, were avoided by requiring $>10$ published reports of adverse reaction (similar studies have only required 2 reports as the threshold) in addition to PRR or EBGM $>2$. By using two separate statistical methods, we minimized false negatives, however, both systems failed to detect several drugs, including protamine or nitroglycerine, with well established relationships to infusion reactions [42]. Reactions caused by these drugs were possibly underreported because the effects have been long known. Furthermore, if the nomenclatures for a certain drug were poor or inaccurate, there could have been missed molecular targets and/or mechanisms.

\section{Conclusions}

Infusion reactions represent drug hypersensitivity with a spectrum of variability and heterogeneity for both individual and drug. The spectrum of symptoms, variable onset that is sometimes rate-related, as well as the ontologic complexity surrounding infusion reactions increases the need for statistical and software modeling that can simultaneously query multiple databases. Using the two statistical methods PRR and EBGM, we maximized specificity in an attempt to preserve sensitivity. Mechanisms for these infusion reactions appear to have significant overlap and implicate NO signaling as a common 
Citation: Moore PW, Burkhart KK, Jackson D (2014) Drugs Highly Associated with Infusion Reactions Reported using Two Different Data-mining Methodologies. J Blood Disorders Transf 5: 195. doi: 10.4172/2155-9864.1000195

pathway.

\section{Author's Contributions}

PM is employed by Pinnacle Health and is the guarantor of the paper and takes responsibility for the integrity of the work. PM queried the MASE and FAERS databases with $\mathrm{KB}$ who is employed by the Food and Drug Administration. DJ, employed by Molecular Health, assisted with manuscript preparation and editing as well as reproducing dependency graphs from MASE.

\section{Acknowledgement}

The authors thank Helen Houpt MSLS AHIP (Pinnacle Health Library Services) for assistance with editing and manuscript preparation and Elizabeth Morgan MLS (Pinnacle Health Library Services) for retrieving references.

\section{References}

1. Dillman RO (1999) Infusion reactions associated with the therapeutic use of monoclonal antibodies in the treatment of malignancy. Cancer Metastasis Rev 18: $465-471$.

2. Miebach E (2009) Management of infusion-related reactions to enzyme replacement therapy in a cohort of patients with mucopolysaccharidosis disorders. International journal of clinical pharmacology and therapeutics 47 : S100-106.

3. Pakravan N, Waring WS, Sharma S, Ludlam C, Megson I, et al. (2008) Risk factors and mechanisms of anaphylactoid reactions to acetylcysteine in acetaminophen overdose. Clin Toxicol (Phila) 46: 697-702.

4. Sandilands EA, Bateman DN (2009) Adverse reactions associated with acetylcysteine. Clin Toxicol (Phila) 47: 81-88.

5. Greenberger PA, Ditto AM (2012) Anaphylaxis. Allergy and asthma proceedings : the official journal of regional and state allergy societies 33: S80-83.

6. Masson MJ, Collins LA, PohI LR (2010) The role of cytokines in the mechanism of adverse drug reactions. Handb Exp Pharmacol: 195-231.

7. Suntharalingam G, Perry MR, Ward S, Brett SJ, Castello-Cortes A, et al (2006) Cytokine storm in a phase 1 trial of the anti-CD28 monoclonal antibody TGN1412. N Engl J Med 355: 1018-1028.

8. Winkler U, Jensen M, Manzke O, Schulz H, Diehl V, et al. (1999) Cytokinerelease syndrome in patients with B-cell chronic lymphocytic leukemia and high lymphocyte counts after treatment with an anti-CD20 monoclonal antibody (rituximab, IDEC-C2B8). Blood 94: 2217-2224

9. Carrico JA, Sabat AJ, Friedrich AW, Ramirez M, et al. (2013) Bioinformatics in bacterial molecular epidemiology and public health: databases, tools and the next-generation sequencing revolution. Euro surveillance 18: 20382.

10. Hauben M, Horn S, Reich L (2007) Potential use of data-mining algorithms for the detection of 'surprise' adverse drug reactions. Drug Saf 30: 143-155.

11. Szarfman A, Tonning JM, Doraiswamy PM (2004) Pharmacovigilance in the 21st century: new systematic tools for an old problem. Pharmacotherapy 24 : 1099-1104.

12. Hauben M (2004) Early postmarketing drug safety surveillance: data mining points to consider. Ann Pharmacother 38: 1625-1630.

13. Hauben M (2004) Application of an empiric Bayesian data mining algorithm to reports of pancreatitis associated with atypical antipsychotics. Pharmacotherapy 24: $1122-1129$

14. Hauben M, Reich L (2004) Data mining, drug safety, and molecula pharmacology: potential for collaboration. Ann Pharmacother 38: 2174-2175.

15. Kadoyama K, Kuwahara A, Yamamori M, Brown JB, Sakaeda T (2011) Hypersensitivity reactions to anticancer agents: data mining of the public version of the FDA adverse event reporting system, AERS. Journal of experimental \& clinical cancer research 30: 93

16. Hauben M, Reich L (2004) Safety related drug-labelling changes: findings from two data mining algorithms. Drug Saf 27: 735-744.

17. Bailie GR, Hörl WH, Verhoef JJ (2011) Differences in spontaneously reported hypersensitivity and serious adverse events for intravenous iron preparations: comparison of Europe and North America. Arzneimittelforschung 61: 267-275.

18. Nakamura T, Lipton SA (2013) Emerging role of protein-protein transnitrosylation in cell signaling pathways. Antioxidants \& redox signaling 18: 239-249.

19. Pacher P, Beckman JS, Liaudet $L$ (2007) Nitric oxide and peroxynitrite in health and disease. Physiol Rev 87: 315-424.
20. Pawloski JR, Hess DT, Stamler JS (2001) Export by red blood cells of nitric oxide bioactivity. Nature 409: 622-626.

21. Toledo JC Jr, Augusto O (2012) Connecting the chemical and biological properties of nitric oxide. Chem Res Toxicol 25: 975-989.

22. Hong YB, Kim EY, Jung SC (2006) Upregulation of proinflammatory cytokines in the fetal brain of the Gaucher mouse. J Korean Med Sci 21: 733-738.

23. Saban-Ruiz J, Alonso-Pacho A, Fabregate-Fuente M, de la Puerta GonzalezQuevedo C (2013) Xanthine oxidase inhibitor febuxostat as a novel agen postulated to act against vascular inflammation. Anti-inflammatory \& antiallergy agents in medicinal chemistry 12: 94-99.

24. Torfgård KE, Ahlner J (1994) Mechanisms of action of nitrates. Cardiovasc Drugs Ther 8: 701-717.

25. Chan ED, Pott GB, Silkoff PE, Ralston AH, Bryan CL, et al. (2012) Alpha-1antitrypsin inhibits nitric oxide production. J Leukoc Biol 92: 1251-1260.

26. Kerr F, Dawson A, Whyte IM, Buckley N, Murray L, et al. (2005) The Australasian Clinical Toxicology Investigators Collaboration randomized trial of different loading infusion rates of $\mathrm{N}$-acetylcysteine. Ann Emerg Med 45: 402-408.

27. Horowitz BZ, Hendrickson RG, Pizarro-Osilla C (2006) Not so fast! Ann Emerg Med 47: 122-123.

28. Joerger $M$ (2012) Prevention and handling of acute allergic and infusion reactions in oncology. Ann Oncol 23 Suppl 10: 313-319.

29. Chung $\mathrm{CH}$, Mirakhur B, Chan E, Le QT, Berlin J, et al. (2008) Cetuximabinduced anaphylaxis and IgE specific for galactose-alpha-1,3-galactose. $\mathrm{N}$ Engl J Med 358: 1109-1117.

30. Kimby E (2005) Tolerability and safety of rituximab (MabThera). Cancer Treat Rev 31: 456-473.

31. Nossaman B, Pankey E, Kadowitz P (2012) Stimulators and activators of soluble guanylate cyclase: review and potential therapeutic indications. Crit Care Res Pract 2012: 290805.

32. Sampson HA, Munoz-Furlong A, Campbell RL, Adkinson NF, Bock SA, et al. (2006) Second symposium on the definition and management of anaphylaxis: summary report--Second National Institute of Allergy and Infectious Disease/ Food Allergy and Anaphylaxis Network symposium. The Journal of allergy and clinical immunology 117: 391-397.

33. Lowery MM, Greenberger PA (2003) Amphotericin-induced stridor: a review of stridor, amphotericin preparations, and their immunoregulatory effects. Annals of allergy, asthma \& immunology: official publication of the American College of Allergy, Asthma, \& Immunology 91: 460-466.

34. Lantoine $F$, louzalen L, Devynck MA, Millanvoye-Van Brussel E, David-Dufilho M (1998) Nitric oxide production in human endothelial cells stimulated by histamine requires Ca2+ influx. Biochem J 330 : 695-699.

35. Renz CL, Laroche D, Thurn JD, Finn HA, Lynch JP, et al. (1998) Tryptase levels are not increased during vancomycin-induced anaphylactoid reactions. Anesthesiology 89: 620-625.

36. Xi L, Zhu SG, Hobbs DC, Kukreja RC (2011) Identification of protein targets underlying dietary nitrate-induced protection against doxorubicin cardiotoxicity. $\mathrm{J}$ Cell Mol Med 15: 2512-2524.

37. Koppenol WH, Moreno JJ, Pryor WA, Ischiropoulos H, Beckman JS (1992) Peroxynitrite, a cloaked oxidant formed by nitric oxide and superoxide. Chem Res Toxicol 5: 834-842.

38. Mullins DW, Alleva DG, Burger CJ, Elgert KD (1997) Taxol, a microtubulestabilizing antineoplastic agent, differentially regulates normal and tumorbearing host macrophage nitric oxide production. Immunopharmacology 37 : $63-73$.

39. Decorti G, Bartoli Klugmann F, Candussio L, Baldini L (1996) Effect of paclitaxe and Cremophor EL on mast cell histamine secretion and their interaction with adriamycin. Anticancer Res 16: 317-320.

40. Lieberman P (2006) Anaphylaxis. Med Clin North Am 90: 77-95.

41. Scarlet $C$ (2006) Anaphylaxis. Journal of infusion nursing: the official publication of the Infusion Nurses Society 29: 39-44.

42. Viaro F, Dalio MB, Evora PR (2002) Catastrophic cardiovascular adverse reactions to protamine are nitric oxide/cyclic guanosine monophosphate dependent and endothelium mediated: should methylene blue be the treatment of choice? Chest 122: 1061-1066 